\title{
Research at the National Bureau of Standards Appli- cable to Long-Distance Location and Direction-Finding Problems ${ }^{1,2}$
}

\author{
R. Silberstein
}

(September 8, 1960; revised December 30, 1960)

\begin{abstract}
Research pertaining to radio-location and direction-finding problems conducted at the National Bureau of Standards since 1941 includes evolution of a technique for determining polarization error, study of non-great-circle bearings, the development of a rapidscanning directional antenna, and the development of Loran-C with its precision timing capabilities.
\end{abstract}

\section{Introduction}

Work in long-distance radio-location and direction finding at the National Bureau of Standards began in the Radio Section in Washington, D.C., which became the Interservice Radio Propagation Laboratory (IRPL) during World War II and later became the Central Radio Propagation Laboratory (CRPL) now located at Boulder, Colorado.

\section{Polarization Error Studies During World War II}

A direction finder may be responsive to vertical or horizontal polarization or to both polarizations phased in various ways, but one polarization may produce an undesired response which causes an error in the reading. This undesired response is a function of the geometrical properties of the instrument, its mountings and various accessories, such as RF cables. A combination of modes in the downcoming ionospheric wave results in more or less random polarization. From instant to instant the relative amplitudes and phases of the wanted and unwanted components of the arriving wave keep changing causing the polarization error to change.

It would be a very difficult thing to compare direction finders and evaluate their polarization errors by observing sky waves, for which bearings depart from their true great circle values for a variety of causes. Some simple test technique was desired which would offer an estimate of the polarization error propensities of any particular direction-finder design. The first test of this type had been evolved by Barfield in England. A balloon with a target transmitter and antenna producing a circularly-polarized wave at a downcoming angle of $45^{\circ}$ was placed near the equipment under test. The polarization error observed was called the "standard wave error."

1 Contribution from Central Radio Propagation Laboratory, National Bureau of Standards, Boulder, Colo.

Paper presented at the Conference on Transmission Problems Related to High-Frequency Direction Finding, at UCLA, June 21-24, 1960.
At NBS, a group under the late Harry Diamond devised techniques whereby pickup factors for the desired and undesired polarizations were determined from fixed target transmitters. These pickup factors were then treated analytically in accordance with a procedure designed for each type of direction finder and polarization errors determined for any set of conditions.

In the case of the simple Adcock antenna, the desired polarization is parallel to the plane of propagation (vertical when the downcoming angle is zero) and the undesired polarization is perpendicular to the plane of propagation (horizontal).

The pickup factor for a wave polarized parallel to the plane of propagation was called $h$. That for a wave polarized perpendicularly to the plane of propagation was called $k$. If $h$ and $k$ were known then the polarization error could be written (in the notation used by the Diamond group) as:

$$
\tan \epsilon=\frac{k}{h} \frac{E_{\perp}}{E_{\| z} \cos \psi}
$$

where

$\epsilon$ is the polarization error angle,

$E_{\perp}$ is the resultant total field vector perpendicular to the plane of propagation (i.e., horizontal polarization) after combination of the incident and ground reflected wave,

$E_{\| z}$ is the resultant total field vector normal to the ground (vertically polarized) after the combination of the ground wave and ground-reflected wave polarized parallel to the plane of propagation $\psi$ downcoming angle

A figure of merit was proposed for the case of equal resultant fields with the direction finder on the ground. Here $\tan \epsilon_{o}=(k / h)$, either $k / h$ or $\epsilon_{o}$ being the figure of merit.

The advantage of the method was that $h$ and $k$ could be measured on the ground. One type of error called "radiator parallax" occurred in measuring $k$ in direction finders like the balanced $\mathrm{H}$ Adcock. 
This was due to a net unwanted vertical pickup in the system when a horizontal dipole was used as a source of perpendicular polarization. In the case of spaced vertical loops another type of error called "collector parallax" was studied. The forward tilt component of the target transmitter's magnetic vector produced an unbalanced voltage in the system. Each type of error could be minimized by increasing the distance to the target transmitter.

The tests for determining $h$ and $k$ were actually conducted with both the direction finder and the target transmitter on wooden platforms above ground. The elevated platforms were especially useful in obtaining a strong horizontally-polarized ground wave for measuring $k$. The importance of this arrangement is attested to by the author, who at one time attempted to measure the horizontal field strength over a stretch of swamp where vertical polarization was attenuated about $70 \mathrm{db}$ less than horizontal. The transmitting antenna was a wire dipole. Minor unbalances of this antenna as it swayed in the breeze produced great surges of output in the field strength meter as incidental pickup of the vertical polarization responded to the vertical component of the unbalanced antenna system.

\section{Radio Propagation Effects on High Fre- quency Direction Finders (World War II Period)}

In this project several receiving stations took routine bearings on distant stations. Results were compared with predicted path MUF's and field strengths. Much attention was paid to an old phenomenon which was noted by the British in the 1930's [Keen, 1947] and which has recently been attracting attention again, namely, propagation of signals by a quasi-scattered non-great-circle mode after failure of the regular path MUF.

The British results were duplicated again in various ways during World War II. For instance, when a BBC broadcast beamed at North Africa was monitored in Virginia, the bearings taken during the hours of predicted MUF above the operating frequency indicated that the bulk of the received energy was arriving via the great circle path. After predicted MUF failure of the eastern control point, however, the signals exhibited reduced fluctuating field strength with widely swinging bearings suggesting a scattering region in Africa. It was assumed that the MUF was still high enough to the south of England for propagation to the scattering region from which energy was being received in Virginia by regular propagation over a more southerly path. In those days it was assumed, following $\mathrm{T}$. $\mathrm{L}$. Eckersley, that high-frequency backscattering and sidescattering occurred in the $E$ region rather than at the ground where most of it is now known to take place, but the general assumption of propagation from a scattering region was valid.

Frequently European stations in the lower part of the high-frequency band, from which great-circle propagation via the regular layers was not possible, were observed to have a northerly bearing deviation when field strength was weak, suggesting a mode involving an Auroral Zone gradient. These studies are called to mind by the results of recent Canadian experiments on $40 \mathrm{Mc} / \mathrm{s} \mathrm{BBC}$ television in which both types of bearing deviations were seen.

Similar observations of signals from United States stations were readily explained, but a group of widely deviated bearings of a Singapore station, observed at Sterling, Va., were never fully understood.

\section{Recent Developments in Techniques}

A precise system of direction finding, developed for use on spheries at VLF, has potentialities for other uses [Hefley, Linfield, and Davis, 1961]. The name "Ephi" was chosen for the system because the bearing of the transient signal is determined from the relative phase (phi) of the vertical electric field (E) received at spaced antennas. In the system three vertical antennas are erected at the corners of an equilateral triangle of sides $1 / 5$ to $1 / 10$ wavelength. A transient signal from a given direction arrives at each antenna at a different time. These arrival times are not compared directly; instead, sets of fixed time delays are inserted in each channel, each set defining an azimuthal sector. Whenever a spheric occurs in such a sector the pulses arrive nearly simultaneously at the outputs of their respective channels and trigger a coincidence circuit. Thus the number of spherics in any sector may be counted. The system is superior to the conventional crossed-loop system in that it works on only vertical polarization. Also it lends itself to automatic readout much more readily than a system employing oscilloscopes.

Testing of a new type of wide-aperture direction finder using electronic scanning has just been completed at CRPL [Cottony and Wilson, 1961]. The model tested had a beamwidth of $6 \%^{\circ}$ and scanned over an arc of $45^{\circ}$. Although the present operating frequency is near $40 \mathrm{Mc} / \mathrm{s}$ special application of the basic features to high frequency work appears to be feasible.

A theoretical paper was written on a proposed experimental technique for measuring the angle of arrival, azimuth and polarization of a downcoming wave by the use of a combined 4-element Adcock and crossed-loop direction finder [Wait, 1959].

\section{Loran C as a Source of Precise Timing}

A system of Loran stations on $100 \mathrm{kc} / \mathrm{s}$, known as the East Coast chain has recently been installed with a master station at Cape Fear, N.C., and two slave stations at Martha's Vineyard, Mass., and Jupiter Inlet, Fla. [Doherty, Hefley, and Linfield, 1961]. These stations employ a recently declassified system of navigation formerly known as Cytac.

In Loran $\mathrm{C}$ the difference between the differentiated signal envelope and the signal itself yields a wave crossing the zero axis at a point so well-defined that it can be used to eliminate ambiguity of a single cycle 
in timing a point on the wave. Synchronization is accomplished entirely by computer circuits.

A slave station can be held to $0.1 \mu$ sec or better. The timing accuracy using the Loran $\mathrm{C}$ ground-wave emissions is $1 \mu \mathrm{sec}$ or better over land for ranges to about 1,500 miles and $0.1 \mu$ sec over sea for ranges to about 2,000 miles. Much greater distances can be covered by sky-wave synchronization with errors of only a few microseconds. Because of the sporadic impulsive nature of atmospheric noise and the fact that the Loran $\mathrm{C}$ pulses arrive at a 20-cycle per second rate, it is possible to synchronize oscillators with a 40 $\mathrm{db}$ ratio of average noise power to signal power.

The advantage of a positioning system with an accuracy 1,000 times greater than that for independent clocks compared with WWV sky-wave signals (assuming a 1-min integration time) is obvious.

\section{References}

Cottony, H. V., and A. C. Wilson, A high-resolution rapid-scan antenna, to be published in J. Research NBS 65D (Radio Prop.) (1961).

Doherty, R., G. Hefley, and R. F. Linfield, Timing potentials of Loran C, to be published in Proc. IRE 1961.

Hefley, G., R. F. Linfield, and T. L. Davis, The Ephi system for VLF direction finding, J. Research NBS 65C (Eng. and Instr.) No. 1, 43 (Jan.-March 1961).

Keen, R., Wireless direction finding, pp. 410-415 (Iliffe \& Sons, 4th ed., 1947).

Wait, J. R., Downcoming radio waves, measurement of characteristics, Electronic \& Radio Engineer 36, No. 3, pp. 106-107 (March 1959). 